\title{
Analysis of the purge time values for pressurized enclosure using a parameterized CFD model
}

\author{
Danut Grecea ${ }^{1 *}$, Gabriela Pupazan ${ }^{1}$, Zoltan Vass ${ }^{1}$, Cosmin Colda $^{1}$, and Adriana Andris ${ }^{1}$ \\ ${ }^{1}$ National Institute for Research and Development in Mine Safety and Protection to Explosion - \\ INSEMEX, 32-34 G-ral Vasile Milea Street, Petrosani, Romania
}

\begin{abstract}
The use of computerized simulations offers definite advantages in understanding phenomena and analysing parameters that have influence during a complex process, more can provide on the basis of scenarios valuable information on the effects for physical processes analysed. Thus, in this paper, using mathematical models and numerical solutions based on physical principles and functions with assumed accuracy are analyse, using CFD (Computational fluids dynamics), the values of purge time for pressurized enclosure, resulting in the action of post-processing of construction for graphs and colour icons, in order to render images as representative possible both for the design phase of the equipment with protection type pressurization " $p$ " and for their evaluation for certification. Determining the values of purging time by using computer simulations is particularly important for avoiding catastrophic events caused by explosions that can lead to human casualties, significant material losses or have significant environmental consequences.
\end{abstract}

\section{Introduction}

The use of simulation programs to understand some phenomena (mode of concentration distribution, concentration dilution) and determine the essential parameters is common in all industries regardless for nature of the services provided. Computer simulations are used both in the design phase, transport production and in the operation phase to optimize processes on the one hand but also to discover, based on well-argued scenarios, the effects in case of rare or unlikely events. Thus, in this paper is presented the concrete case of use a simulation, using a specialized software, for determination and analysis of the time required to purging a pressurized enclosure.

\subsection{Pressurized enclosure protection type "p" used in classified areas}

The occurrence of an explosion causes significant material damage but above all can cause human losses that cannot be quantified, so the assessment of the risk of explosion and establishment of appropriate measures to reduce for acceptable levels in accordance with

\footnotetext{
*Danut Grecea : danut.grecea@insemex.ro
} 
current standards and regulations are particularly important in view of increase in human health and safety but also the protection of surrounding environment [1].

Explosive atmospheres are mixtures of flammable substances, in the form of fine dispersion of gases/ vapours/ conductive dust with air, under normal atmospheric conditions, for which the ignition burns violently (explosive) from source in entire volume of mixture [2]. Thus, the existence of flammable substance in atmosphere, followed by its ignition, can produce a wide variety of thermal and dynamic effects [3].

Explosion prevention and explosion protection are of major importance and to minimize the risk of explosion all equipment operating in explosive atmospheres must be designed so as not to ignite the explosive atmospheres for which they are designed to operate [4].

All constructive solutions applied to electrical or non-electrical equipment that are used in Ex environments are types of protection. Constructively, electrical equipment may have several types of protection specified by manufacturer on label, according to requirements of applicable standards, and each type of protection is based on a technical solution for implementation of explosion protection. The technical solution with highest incidence for the implementation of explosion protection is based on separation of hazardous atmosphere from the parts of equipment, which can become a source of ignition. One such solution is to protect the equipment by pressurized enclosure [5].

The principle of the protection type trough pressurized enclosure " $p$ " consists in separating potentially explosive atmosphere from ignition source by maintaining an overpressure inside enclosure with help of a protection gas (air or inert gas) [6].

Thus, this type of protection has three levels of protection (pxb, pyb and pzc) which are chosen according to the level of protection of the equipment (EPL) required for an external explosive atmosphere ( $\mathrm{Mb}, \mathrm{Gb}$ or $\mathrm{Gc})$, if there is internal release and if the equipment / components inside the pressurized housing can produce ignition and can be used in zone 1 or 2, fulfilling the requirements for equipment categories 2 or 3, (Figure 1) [7].

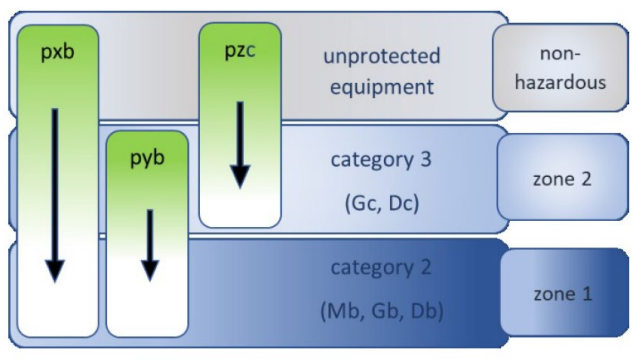

Fig. 1. Correlation between protection levels "p" and the equipment category/ corresponding area.

\subsection{Evaluation of filling and purging time using computer simulation}

By approaching a gloomy scenario of explosion by simulating and analysing dispersion of dangerous substances (explosive) released into environment, the consequences can be highlighted but especially possible measures to avoid consequences and minimize the effects. The simulation can be used both to design equipment, to analyse essential parameters on which depends protection type and their limitations in extreme conditions but, unfortunately and later after occurrence of events to understand event and find solutions, implementing measures to increase level of occupational safety and environmental protection.

A software used in analysis of phenomena generated by explosions and their consequences is CFD software package. CFD (Computational Fluids Dynamics) used to perform computer simulations, like in this paper, is an application for different stages and is an integral part of ANSYS Multiphysics package. This CFD package is a comprehensive tool 
for Multiphysics analysis, which allows the user to combine effect of two or more physical phenomena (structural, electrical, thermal, electromagnetic, magnetic, fluid flow, electrostatic).

The following applications were used in simulation:

- ANSYS Design Modeler - contains modelling tools specific to simulation requirements, including: tools for creating geometric model and possibility to modify the existing geometry.

- ANSYS Meshing - realizes discretization of virtual geometries and contains very complex pre/ post - processing tools, with direct connection to solvents and offers a direct path from CAD programs to pre/ post CAE processing.

- ANSYS FLUENT - is a flexible CFD application, used for simulations of any complexity. It offers a full range of physical models that can be used for a wide range of applications in various industries [8].

\section{Simulation method}

The certification process of equipment used in Ex environments involves in addition to equipment evaluation and testing to demonstrate practical compliance with requirements set by specific standard [4 -6].

Depending on the pressurization method chosen for equipment, certain type tests shown synthetically in Figure 2.

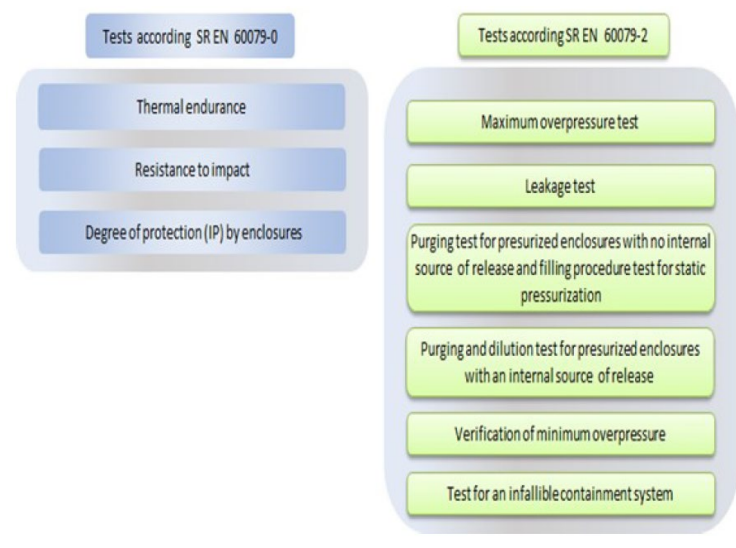

Fig. 2. Tests for equipment protected by pressurized enclosure.

In In addition to required tests, test gases and devices for measuring the concentration inside the enclosure must use for the filling test followed by purging test (presented in this paper and are performed by simulation).

The standard requires that at filling tests all points inside pressurized enclosure of equipment must have a concentration ratio of over $70 \%$ volume/ volume. In the same way, for purge tests, it is necessary that all internal points of enclosure for protected equipment have a concentration ratio below $0.25 \%$ or $1 \%$ volume/ volume, depending on the test gas used (carbon dioxide or helium) $[6,7,9]$.

The construction of interior space of pressurized equipment has an important role in filling process or purging, so for certain areas, the time to reach the desired value of concentration is longer than for other areas. For simple pressurized enclosure, position of critical points can be found intuitively, given relative density of test gas relative to air, but for other cases these points should be explored, activity that consumes time and gas, but especially does not provide certainty that critical points are accurately identified. But for 
these particular cases, simulation using fluid dynamics and calculation programs is particularly important and useful [9].

In study, presented in the paper, through mathematical models and numerical solutions based on physical principles and functions with assumed accuracy are analysed, using CFD (Computational fluids dynamics), values of filling time and subsequent purging of a pressurized enclosure. Following the simulations performed from CFD program, the mechanical effects can be determined in ensure correlation between analysed times in relation to position of the studied points.

CFD applications are tools for computer simulation of physical processes through mathematical models, resulting in values of selected variables that, through post-processing, contribute to construction of graphs, vector fields, colour icons, to give the user images as representative of studied phenomena. All these, gathered behind the CFD application, represent the so-called Blackbox. The user enters input data, which is processed in Blackbox, after which the results are issued. Entering erroneous input data will lead to erroneous results. The CFD software package includes modules for creating geometric spaces, building discretization networks, flow simulation and post-processing. Solving problems using CFD techniques involves defining several steps, illustrated in Figure 3, this structure being used in the case of simulation to determine filling and purging time for case analysed [8, $10-11]$.

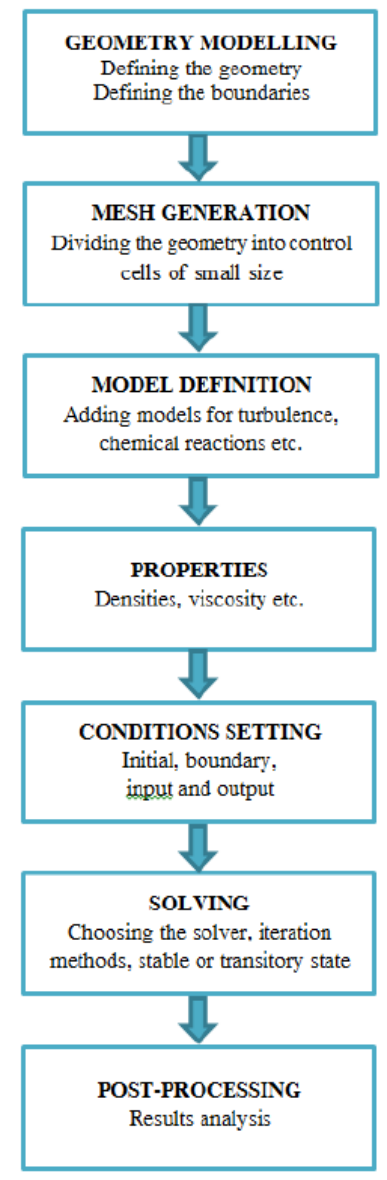

Fig. 3. Steps required for CFD simulation. 


\section{Results and discussion}

For simulation, analysis and evaluation of filling and purging times in certain points of interest, a pressurized enclosure with the following dimensions $400 \times 300 \times 600 \mathrm{~mm}$ and supply hole with a diameter of $10 \mathrm{~mm}$ is considered, and analysed points are located according to Figure 4.
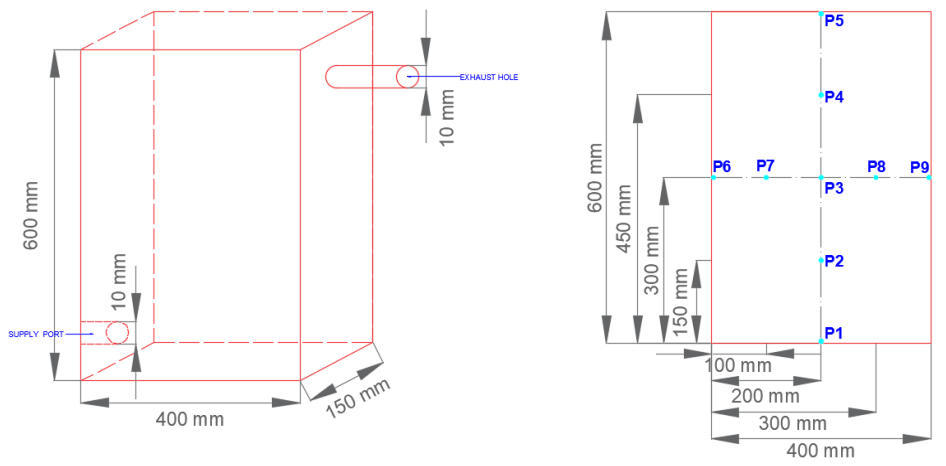

Fig. 4. Dimensions of the pressurized enclosure and location for analysed points.

Time required to simulate filling and purging of pressurized enclosure, so that the analysed points meet specific standard [6], is $165 \mathrm{~s}$, Figure 5.

Use procedure for simulation process is presented as follows:

- initially, entire enclosure has $100 \%$ air in all interior points;

- $\mathrm{CO}_{2}$ is introduced on inlet port at an overpressure of $1000 \mathrm{~Pa}$. Filling with $\mathrm{CO}_{2}$ is continued until volume ratio is at least $70 \%$ volume/ volume $/ \mathrm{CO}_{2}$ for all points analysed;

- necessary time is recorded (filling time being in the range of $0 \mathrm{~s}$ to $79 \mathrm{~s}$ );

- after filling operation, is purging with air at the same overpressure. Air purge is continued until volume ratio is at least $0.25 \%$ volume/ volume/ $\mathrm{CO}_{2}$ at all points studied;

- required time is recorded (purging time being in the range of $81 \mathrm{~s}$ to $162 \mathrm{~s}$ ).

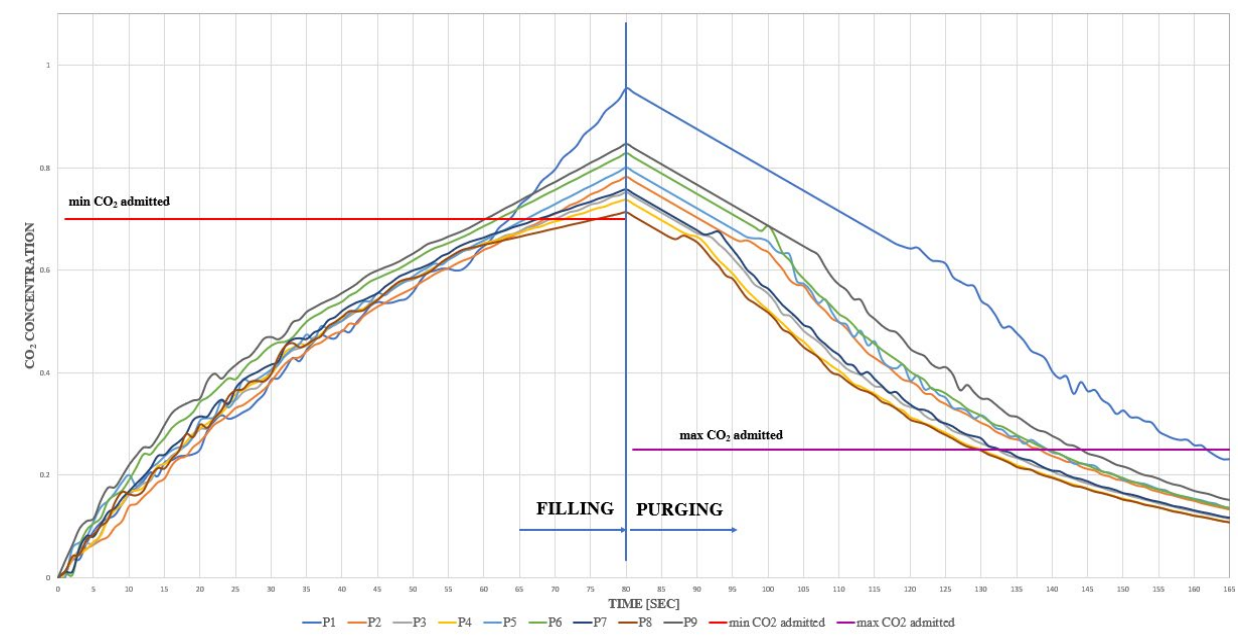

Fig. 5. Variation of filling and purging time in analysed points. 
For filling operation, the analysed elements, resulting from simulation are presented in Figures $6-8$.

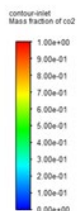

Time :

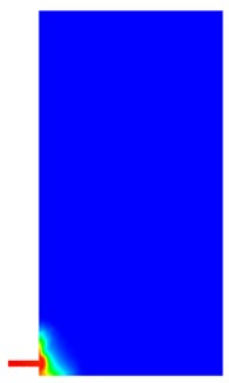

$2[\mathrm{~s}]$

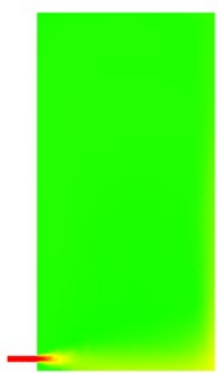

$38[\mathrm{~s}]$

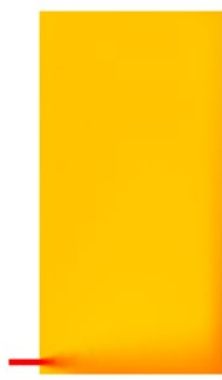

$73[\mathrm{~s}]$

Fig. 6. Variation of $\mathrm{CO}_{2}$ concentration during filling procedures.

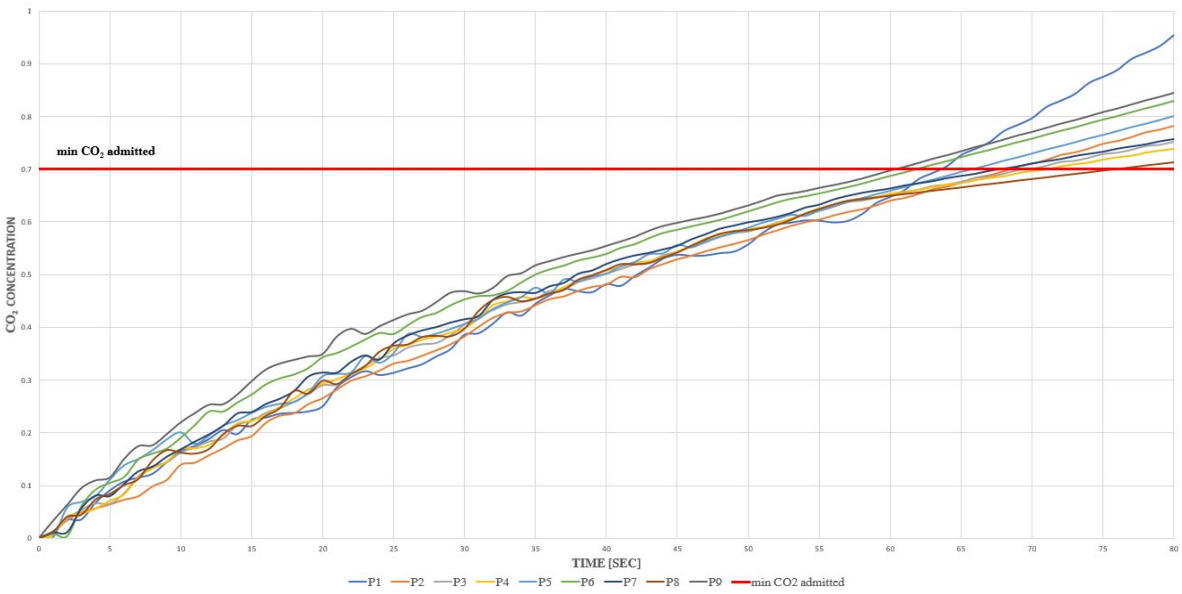

Fig. 7. $\mathrm{CO}_{2}$ concentration and filling time variation in analysed points.

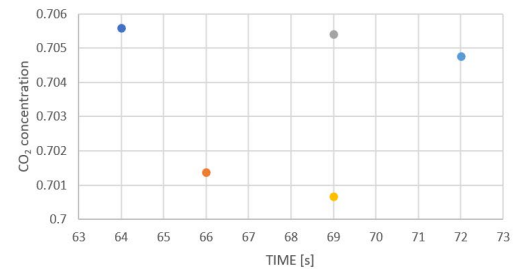

$\bullet \mathrm{P} 1_{\mathrm{P}} \bullet \mathrm{P} 5 \mathrm{P} 2_{\mathrm{P}} \bullet \mathrm{P} 3 \bullet \mathrm{P} 4$

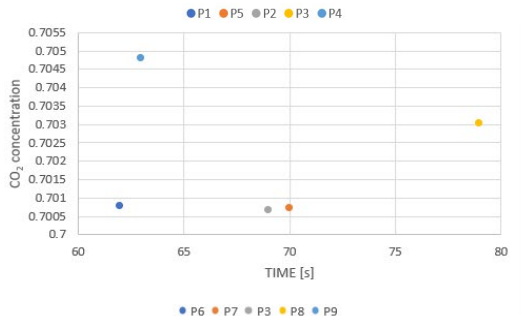

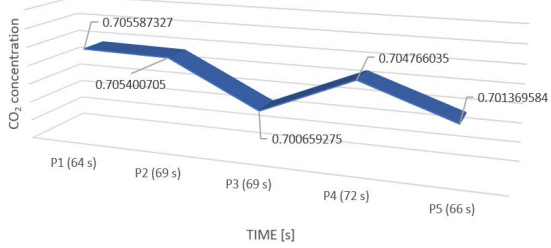

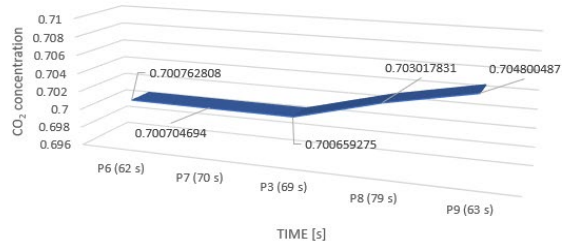

Fig. 8. Time required to reach all points at minimum allowable of $\mathrm{CO}_{2}$ concentration. 
For purge operation in analysed points, the simulation results are presented in Figures 9 11.

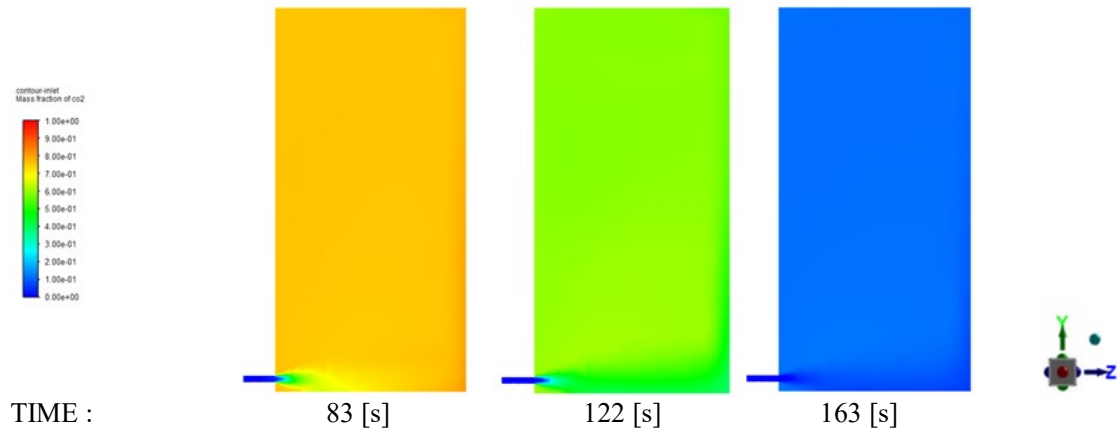

Fig. 9. Variation of $\mathrm{CO}_{2}$ concentration during purging process.

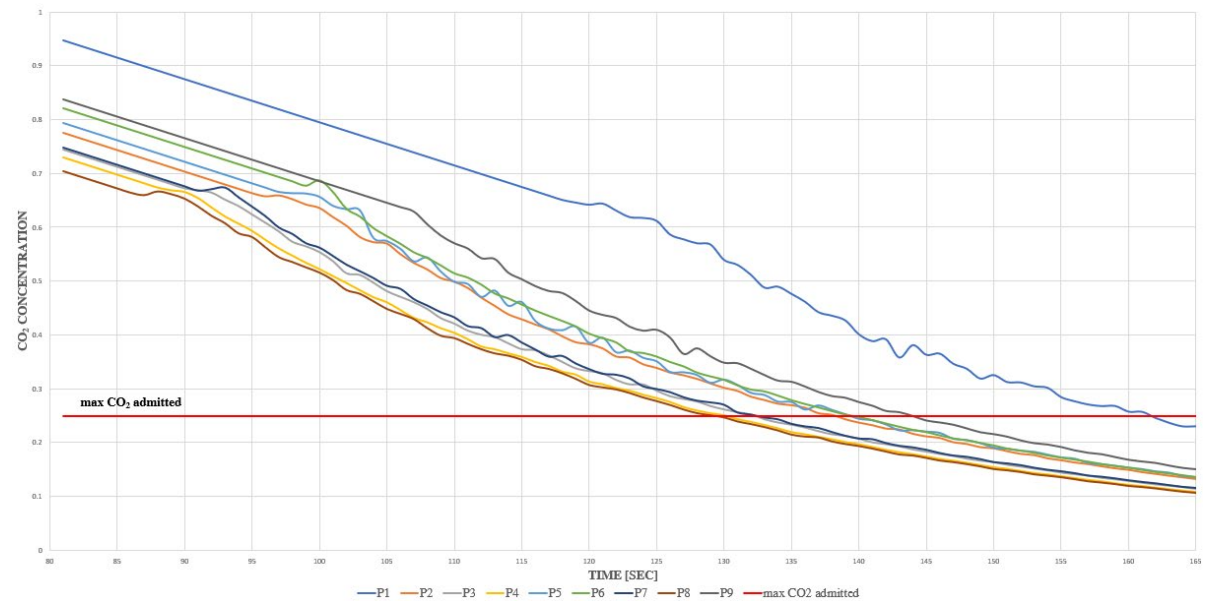

Fig. 10. $\mathrm{CO} 2$ concentration and purge time variation in analysed points.
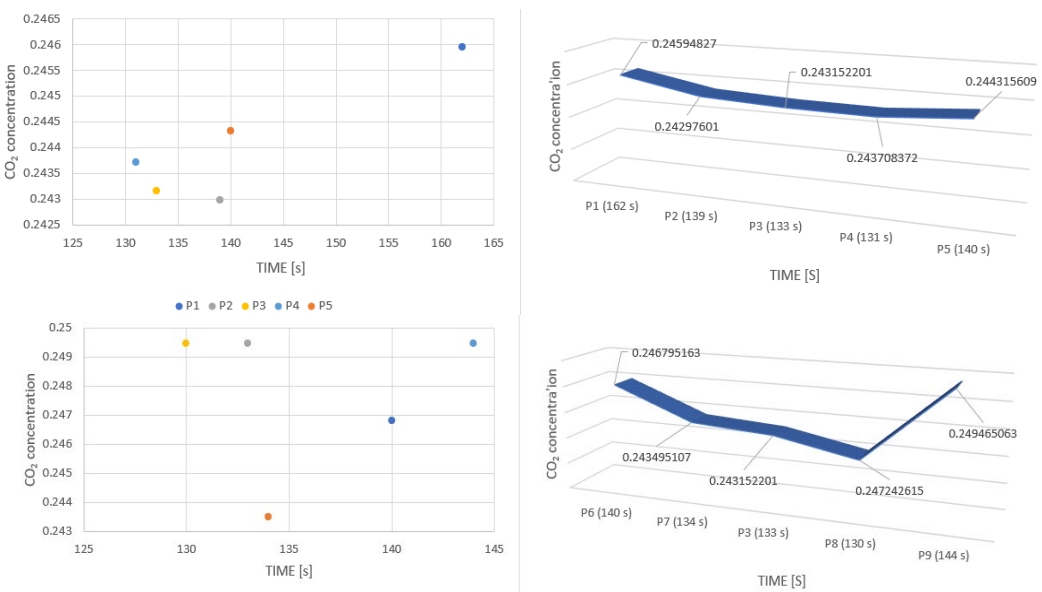

Fig. 11. Time required to reach all points at maximum permissible $\mathrm{CO}_{2}$ concentration. 


\section{Conclusions}

Simulation with CFD utility method is becoming increasingly used in modelling systems that include fluid flow in many areas. CFD codes make it possible to numerically solve transport of fluids, mass and energy balances in systems with very complicated geometry. The obtained results present special flow and transfer models that are difficult to obtain experimentally or by conventional modelling methods.

Use of CFD numerical analysis media in research of conformity for equipment's with pressurized enclosure type of protection, allows determination of important parameters such as: pressure distribution and velocity field inside encapsulation; determining the flow lines of current generated by supply and many other parameters (pressure, temperature). This fact allows evaluation of performance for studied equipment without considerable expenses, with minimum effort and maximum operability.

Following the analysis of simulation, resulting:

- at filling operation, maximum time required, for conditions imposed, is identified to P8 (72 s) followed by the points inside encapsulation (P4 - 72 s, P7 - 70 s and P2, P3 - 89 s), due to circular motion, on the edge of enclosure, of gas (CO2) introduced under pressure:

- for purging process, in addition to influence of circular movement are also involves the influence the by higher density of the gas inside encapsulation (CO2) in relation to air used for purging. Thus, maximum time necessary for requirements for purging is at P1 (162 s from start of simulation, $82 \mathrm{~s}$ from start of purging process), followed by points outside the centre, on edge of encapsulation (P9 - 144 s, P5, P6 - 140 s and following with P2 - 139 s).

The simulation shows that, even if enclosure has a symmetry, values of filling and purging time, regarding the internal volume ratio do not show symmetry and also show influence of turbulence created by internal structure on distribution of $\mathrm{CO} 2$ concentration values.

Even if type of pressure protection mentions that, in some cases, purging inner volume with air (five times the inner volume) will guarantee that for all interior points volume ratio thresholds are met, some interior topology structures may exhibit a behaviour unexpected. In this case, an intuitive way to set the position of measuring points is not $100 \%$ accurate.

\section{References}

1. Directive 2014/34/EU (2014)

2. Standard SR EN 60079-10-1 (2016)

3. E. Ghicioi et al, Environ Eng Manag J, 16, 1290-1294, (2017)

4. Standard SR EN 60079-0 (2018)

5. Standard SR EN 60079-14 (2014)

6. Standard SR EN 60079-2 (2015)

7. G. Pupazan et al, MATEC Web of Conferences 305, (2020)

8. C. Colda et al, IOP Conf. Ser.: Earth Environ. Sci. 609, (2020)

9. T. Csaszar et al, Proceedings of the 19th International Multidisciplinary Scientific Geoconference SGEM 2019, 19, 743-750, (2019)

10. N. Vlasin et al, Proceedings of the 16th International Multidisciplinary Scientific Geoconference SGEM 2016, 16, 965-972, (2016)

11. N. Vlasin et al, Proceedings of the 16th International Multidisciplinary Scientific Geoconference SGEM 2013, 13, 563-570, (2013) 